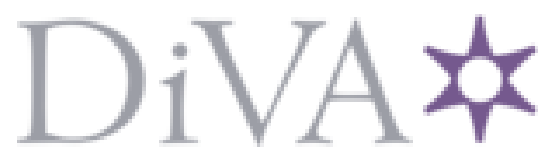

http://www.diva-portal.org

This is the published version of a paper published in Physical Review A. Atomic, Molecular, and Optical Physics.

Citation for the original published paper (version of record):

Dutta, O., Jääskeläinen, M., Meystre, P. (2006)

Thomas-Fermi ground state of dipolar fermions in a circular storage ring.

Physical Review A. Atomic, Molecular, and Optical Physics, 73(4): 043610

http://dx.doi.org/10.1103/PhysRevA.73.043610

Access to the published version may require subscription.

N.B. When citing this work, cite the original published paper.

Permanent link to this version:

http://urn.kb.se/resolve?urn=urn:nbn:se:du-11208 


\title{
Thomas-Fermi ground state of dipolar fermions in a circular storage ring
}

\author{
O. Dutta, M. Jääskeläinen, ${ }^{*}$ and P. Meystre \\ Department of Physics and College of Optical Sciences, The University of Arizona, Tucson, Arizona 85721, USA
}

(Received 25 December 2005; published 18 April 2006)

\begin{abstract}
Recent developments in the field of ultracold gases has led to the production of degenerate samples of polar molecules. These have large static electric-dipole moments, which in turn causes the molecules to interact strongly. We investigate the interaction of polar particles in waveguide geometries subject to an applied polarizing field. For circular waveguides, tilting the direction of the polarizing field creates a periodic inhomogeneity of the interparticle interaction. We explore the consequences of geometry and interaction for stability of the ground state within the Thomas-Fermi model. Certain combinations of tilt angles and interaction strengths are found to preclude the existence of a stable Thomas-Fermi ground state. The system is shown to exhibit different behavior for quasi-one-dimensional and three-dimensional trapping geometries.
\end{abstract}

DOI: $10.1103 /$ PhysRevA.73.043610

PACS number(s): 03.75.Ss, 03.65.Sq, 05.30.Fk

\section{INTRODUCTION}

Exciting new opportunities are now emerging in matterwave optics as a result of the availability of ultracold, possibly quantum-degenerate molecular systems [1-4]. So far, molecular condensation has only been demonstrated when starting from pairs of identical atoms, but two recent experiments have reported the observation of heteronuclear Feshbach resonances in Bose-Fermi mixtures of ${ }^{6} \mathrm{Li}$ and ${ }^{23} \mathrm{Na}$ [5] in one case and of ${ }^{87} \mathrm{Rb}$ and ${ }^{40} \mathrm{~K}$ in the other [6]. Sweeping through such resonances is expected to lead to the coherent formation of a substantial amount of heteronuclear molecules. Alternatively, photoassociation has resulted in the production of ultracold metastable RbCs molecules in their lowest triplet state when starting from a laser-cooled mixture of ${ }^{85} \mathrm{Rb}$ and ${ }^{133} \mathrm{Cs}$ [7].

Much attention has also been given to the trapping and guiding of matter waves in "atom chip" microstructures $[8,9]$, a natural route for applications such as rotation sensors based on the Sagnac effect [10]. Experimental and theoretical work has focused on various trapping schemes for ring geometries, using both magnetic $[11,12]$ and magnetoelectrostatic mechanisms [13]. In several recent developments, Sauer, Barrett, and Chapman [14], Wu et al. [15], and Gupta et al. [11] have realized atomic storage rings in magnetic waveguides. Microtraps for heteronuclear molecules have also been considered by Tscherneck et al. [16]. These molecules [17] are polar, with a permanent electric-dipole moment of the order of $e a_{0}$ [18]. As such, their center-of-mass trajectories can be manipulated by relatively modest static or quasistatic inhomogeneous electric fields [19-21]. The dipole-dipole interaction is central to the dynamics of molecular samples [22-24], where it is expected to lead to fascinating new physics. For example, Bochinski et al. have demonstrated the phase-space manipulation of polar molecules in the presence of a dipole-dipole interaction [25] by using pulsed inhomogeneous electric fields, and it would be of considerable interest to extend such studies to the quan-

\footnotetext{
*Corresponding author. Email address: mrq@optics.arizona.edu
}

tum regime. The anisotropy of the dipole-dipole interaction should also permit us to understand how the nature and stability of quantum-degenerate atomic and molecular systems is influenced by the anisotropic character of interparticle interaction [26]. New kinds of quantum phase transitions have been predicted in these systems [27], and their ground state and stability are expected to differ fundamentally from the situation in condensates characterized by a contact interaction $[26,28]$.

The question that is specifically addressed in the present paper is the role of the dipole-dipole interaction in determining the ground state of quantum-degenerate fermionic polar molecules in a ring-shaped waveguide. By applying a static electric field tilted with respect to the waveguide, the dipoledipole interaction between molecules can be made inhomogeneous along the ring, the amount of inhomogeneity being controlled by the tilt angle. We consider specifically situations where the trapping mechanism is different from the polarizing mechanism, as is possible, for instance, with $\mathrm{CrRb}$ which possesses both a large permanent magnetic-dipole moment and a large static electric-dipole moment.

The paper is organized as follows: Section II discusses the effective dipole-dipole interaction for molecules trapped in a storage ring with circular geometry. In case the transverse trap energy is larger than the mean-field energy associated with the dipole-dipole interaction, the system is quasi one dimensional, while it is truly three dimensional in the opposite limit. Section III concentrates on the quasi-onedimensional case and determines the ground state of the system in the Thomas-Fermi local density approximation. Regions where this approach leads to unphysical results are determined and found to become more of a problem in the regime of low densities, a result of the dominance of the mean-field energy over the kinetic energy. Section IV extends these considerations to the three-dimensional limit, where the transverse distribution is no longer given by the trap ground state. Finally Sec. V is a summary and outlook.

\section{EFFECTIVE INTERACTION}

We consider an ultracold sample of neutral fermionic atoms or molecules with permanent electric- or magnetic- 


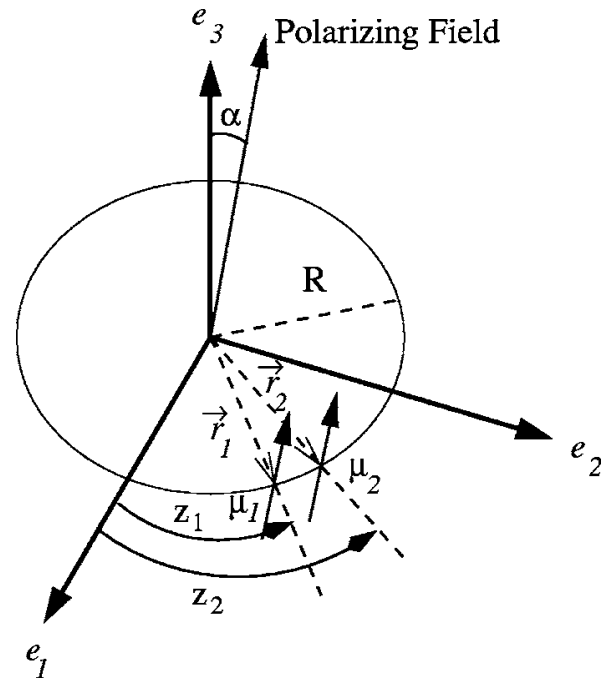

FIG. 1. Storage ring of radius $R$ with dipoles $\mu_{1}$ and $\mu_{2}$ at locations $z_{1}$ and $z_{2}$. The poles are tilted at an angle $\alpha$ with respect to the direction $e_{3}$.

dipole moment $\vec{\mu}$ and confined to a ring-shaped trap of radius $R$ and length $L_{0}$. The interaction between two pointlike dipoles $\vec{\mu}_{1}$, and $\vec{\mu}_{2}$ at positions $\vec{r}_{1}$ and $\vec{r}_{2}$ is given by

$$
V_{\mathrm{dd}}\left(\vec{r}_{1}, \vec{r}_{2}\right)=\frac{1}{4 \pi \varepsilon_{0}}\left[\frac{\vec{\mu}_{1} \cdot \vec{\mu}_{2}}{r^{3}}-\frac{\left(\vec{\mu}_{1} \cdot \vec{r}\right)\left(\vec{\mu}_{2} \cdot \vec{r}\right)}{r^{5}}\right]
$$

where

$$
r \equiv|\vec{r}|=\left|\vec{r}_{1}-\vec{r}_{2}\right|
$$

is the distance between the two dipoles. We consider specifically the situation where the atomic or molecular sample is polarized along some arbitrary direction, in which case Eq. (1) reduces to

$$
V_{\mathrm{dd}}\left(\vec{r}_{1}, \vec{r}_{2}\right)=-\frac{\mu_{1} \mu_{2}}{4 \pi \epsilon_{0} r^{2}}\left[2 \cos ^{2} \theta-\sin ^{2} \theta\right],
$$

where $\theta$ is the angle between $\vec{r}$ and the direction of polarization of the dipoles, which we take to be tilted by an angle $\alpha$ with respect to the normal of the plane of the trap; see Fig. 1.

In the limit of strong fermion confinement in the radial direction, their single-particle wave function of the atoms or molecules can be factorized as

$$
\Psi\left(\vec{r}_{\perp}, z\right)=\Psi_{\perp}\left(\vec{r}_{\perp}\right) \Psi(z),
$$

where $\vec{r}_{\perp}$ is the radial coordinate vector measured from the center of the ring-shaped trap and $z$ is the longitudinal coordinate along the ring. In writing Eq. (4), we implicitly assumed that the transverse trap potential is invariant along $z$. We further assume that the two-particle wave function $\Psi\left(\vec{r}_{1}, \vec{r}_{2}\right)$ can be factorized as

$$
\Psi\left(\vec{r}_{1}, \vec{r}_{2}\right)=\Psi_{\perp}\left(\vec{r}_{1 \perp}\right) \Psi_{\perp}\left(\vec{r}_{2 \perp}\right) \Psi\left(z_{1}, z_{2}\right),
$$

in which case the effective longitudinal dipole-dipole interaction between two identical dipoles at positions $z_{1}$ and $z_{2}$ is given by
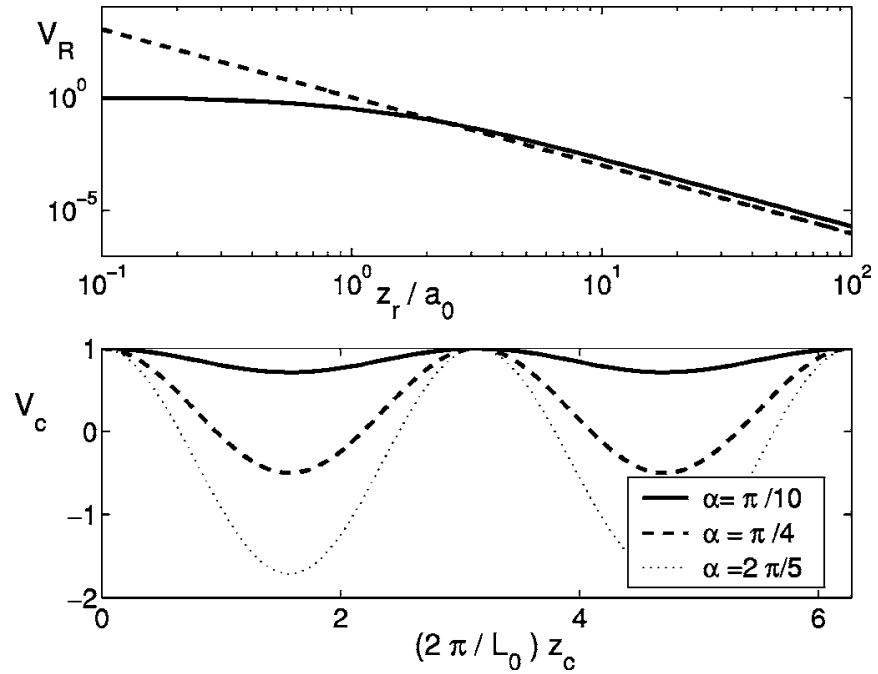

FIG. 2. (a) $V_{r}\left(z_{r}\right)$ as a function of interparticle distance $z_{r}=z_{1}-z_{2}$. (b) $V_{c}\left(z_{c}\right)$ as a function of the center-of-mass coordinate $z_{c}=\left(z_{1}+z_{2}\right) / 2$. In (a) the dashed line shows $\left(z_{r} / a_{0}\right)^{-1 / 3}$, the bare dipole potential, as a reference.

$$
\begin{aligned}
& V_{\mathrm{dd}, \|}\left(z_{1}, z_{2}\right) \\
& \quad=\iint\left|\Psi_{\perp}\left(\vec{r}_{1 \perp}\right)\right|^{2}\left|\Psi_{\perp}\left(\vec{r}_{2 \perp}\right)\right|^{2} V_{\mathrm{dd}}\left(\vec{r}_{1}, \vec{r}_{2}\right) d \vec{r}_{1 \perp} d \vec{r}_{2 \perp} .
\end{aligned}
$$

Assuming that the transverse single-particle wave function $\Psi_{\perp}\left(\vec{r}_{\perp}\right)$ is a Gaussian of half-width $a_{0}$ and that the trap radius $R$ is large compared to the transverse trap size, we can evaluate Eq. (6) to find

$$
\begin{aligned}
V_{\mathrm{dd}, \|}\left(z_{1}, z_{2}\right)= & \frac{\mu^{2}}{4 \pi \epsilon_{0} a_{0}^{3}}\left[3 \sin ^{2} \alpha \sin ^{2}\left(\frac{2 \pi z_{c}}{L_{0}}\right)-1\right] \\
& \times\left[\zeta-\sqrt{\frac{\pi}{2}}\left(1+\zeta^{2}\right) \operatorname{erfcx}\left(\frac{\zeta}{\sqrt{2}}\right)\right],
\end{aligned}
$$

where

$$
\operatorname{erfcx}\left(\frac{\zeta}{\sqrt{2}}\right)=\operatorname{erfc}\left(\frac{\zeta}{\sqrt{2}}\right) \exp \left(-\frac{\zeta^{2}}{2}\right)
$$

and $\operatorname{erfc}(x)$ is the complementary error function. Here, $z_{c}=\left(z_{1}+z_{2}\right) / 2$ and $z_{r}=z_{1}-z_{2}$ are the center-of-mass and relative coordinates of the fermion pair and

$$
\zeta\left(z_{r}\right)=\frac{2 R_{0}}{a_{0}}\left|\sin \left(\frac{\pi z_{r}}{L_{0}}\right)\right| .
$$

The longitudinal potential can therefore be factorized as

$$
V_{\mathrm{dd}, \|}\left(z_{c}, z_{r}\right)=\frac{\mu^{2}}{4 \pi \epsilon_{0} a_{0}^{3}} V_{c}\left(z_{c}\right) V_{r}\left(z_{r}\right) .
$$

The center-of-mass and relative components $V_{c}\left(z_{c}\right)$ and $V_{r}\left(z_{r}\right)$ of the effective potential are plotted in Fig. 2. The short-range behavior of $V_{r}\left(z_{r}\right)$ is regularized by the transverse integration to yield finite values as $z_{r} \rightarrow 0$. For large 
distances, $V_{r}\left(z_{r}\right) \approx z_{r}^{-3}$, as expected for the dipole-dipole interaction. Note also that $V_{c}\left(z_{c}\right)$ depends on the tilt angle $\alpha$ and can become negative for

$$
\alpha>\arcsin \left(\frac{1}{\sqrt{3}}\right)
$$

resulting in the appearance in the storage ring of regions with attractive interaction.

\section{QUASI-ONE-DIMENSIONAL REGIME}

We first consider a quasi-one-dimensional situation where the dipolar fermions are subject to a strong transverse trapping and thus remain in the transverse ground state of the storage ring potential. Our goal is to determine the ground state of the system within the Thomas-Fermi approximation $[29,30]$.

Our starting point is the one-dimensional energy functional

$$
E_{1}=T_{1}+V_{1},
$$

which in the Thomas-Fermi theory can be expressed as a functional of the ground-state particle density distribution. Within that local density approximation, the kinetic energy becomes

$$
T_{1}=\int_{0}^{L} n(z) t(n, z) d z
$$

where $n(z)$ the particle density. The kinetic energy density is

$$
t(n, z)=\int\left(\frac{p_{\|}^{2}(z)}{2 m}\right) \mathcal{P}(p \|(z) d p(z),
$$

where $m$ is the particle mass and $\mathcal{P}\left(p_{\|}(z)\right) d p_{\|}(z)$ is the probability that a particle at the position $z$ has a momentum between $p_{\|}(z)$ and $p_{\|}(z)+d p_{\|}(z)$ and the density dependence is still obscured at this point.

At zero temperature $T=0, \mathcal{P}\left(p_{\|}(z)\right) d p_{\|}(z)$ is given explicitly by

$$
\mathcal{P}\left(p_{\|}(z)\right) d p_{\|}(z)=\left(\frac{1}{2 p_{\|, f}(z)}\right) d p_{\|}(z),
$$

where $p_{\|, f}(z)$ is the position-dependent Fermi momentum. Inserting this expression into Eq. (14) we have

$$
t(n, z)=\frac{p_{\|, f}^{2}(z)}{6 m} .
$$

In one dimension the density $n(z)$ and the Fermi momentum $p_{\|, f}(z)$ are related by

$$
n(z)=\frac{p_{\|, f}(z)}{\pi \hbar} .
$$

Inserting this into Eq. (13) and carrying out the integration results in the Thomas-Fermi kinetic energy functional

$$
T_{1}=\left(\frac{\pi^{2} \hbar^{2}}{6 m}\right) n^{3}(z) .
$$

Combining finally this expression with the interaction energy contribution gives then the Thomas-Fermi energy functional as

$$
\begin{aligned}
E_{1}= & \frac{\pi^{2} \hbar^{2}}{6 m} \int_{0}^{L_{0}} n^{3}(z) d z \\
& +\frac{1}{2}\left(\frac{|\mu|^{2}}{4 \pi \epsilon_{0} a_{0}^{3}}\right) \int_{0}^{L} d z d z^{\prime} V_{\mathrm{dd}, \|}\left(z / a_{0}, z^{\prime} / a_{0}\right) n(z) n\left(z^{\prime}\right),
\end{aligned}
$$

where we have factorized the absolute squared two-body longitudinal wave function as

$$
\left|\Psi\left(z, z^{\prime}\right)\right|^{2}=n(z) n\left(z^{\prime}\right) .
$$

Scaling lengths to the characteristic size $a_{0}$ of the transverse single-particle wave function $\Psi\left(\vec{r}_{\perp}\right)$ via

$$
x=\frac{z}{a_{0}}
$$

and normalizing the density for the correct total number of atoms via

$$
\rho_{1}(x)=\left(\frac{a_{0}}{N}\right) n(z),
$$

with

$$
\int_{0}^{L} \rho_{1}(x) d x=1,
$$

yields finally the dimensionless Thomas-Fermi energy functional as

$$
\varepsilon_{1}=\frac{E_{1}}{\tau N^{3}}=\int_{0}^{L} \rho_{1}^{3}(x) d x+\frac{g_{1}}{2} \int_{0}^{L} d x d x^{\prime} V_{\mathrm{dd}, \|}\left(x, x^{\prime}\right) \rho_{1}(x) \rho_{1}\left(x^{\prime}\right) .
$$

Here $\varepsilon_{1}$ is the dimensionless energy of the trapped fermions and the dimensionless interaction constant $g_{1}$, given by

$$
g_{1}=\frac{1}{\tau}\left(\frac{\mu^{2}}{4 \pi \epsilon_{0} N a_{0}^{3}}\right),
$$

is the ratio of the characteristic transverse dipole-dipole energy to the characteristic transverse kinetic energy per particle:

$$
\tau=\frac{\pi^{2} \hbar^{2}}{6 m a_{0}^{2}} .
$$

The kinetic energy term, which has a cubic dependence on density, dominates over the two-body interaction at high densities. In this quasi-one-dimensional regime the dimensionless interaction strength $g_{1} \propto N^{-1}$ scales in the same way as the dimensionless coupling constant in the Tonks- 
Girardeau gas [31]. Likewise, the scaling of the dimensionless energy (24) is similar to that of the impenetrable Bose gas in the thermodynamic limit [32].

The ground-state density of fermions is obtained by minimizing the energy $\varepsilon$ under the constraint that the total number of particles be equal to $N$. In the variational equation we have

$$
\delta\left[\varepsilon_{1}-\mu_{1}\left(\int_{0}^{L} \rho_{1}(x) d x-1\right)\right]=0,
$$

$\mu_{1}$ being the chemical potential. It should be noted that assuming $\mu_{1}$ to be a variational parameter in the energy functional trivially leads to the conservation of particle number and adds no information about the value of the chemical potential. The resulting integral equation for the variational ground state is

$$
\rho_{1}^{2}(x)+\frac{g_{1}}{3} \int_{0}^{L} V_{\mathrm{dd}, \|}\left(x, x^{\prime}\right) \rho_{1}\left(x^{\prime}\right) d x^{\prime}=\mu_{1} .
$$

Solving Eq. (28) numerically requires knowledge of the chemical potential $\mu_{1}$. In the noninteracting case, it is equal to the Fermi energy, which is given by $1 / L^{2}$ in dimensionless units. For weak interaction strengths, this same value can be taken as a first approximation. The exact value of the chemical potential, however, depends on the interaction strength and, hence, on the degree of inhomogeneity of the system as we shall see.

To determine the longitudinal density profile corresponding to the semiclassical many-body ground state, we invoke the periodicity of the system to express $\rho_{1}(x)$ as a Fourier series in the dimensionless coordinate $x$. It follows from the normalization condition (23) that the first term in such a series must equal $1 / L$, giving

$$
\rho_{1}(x)=\frac{1}{L}\left[1-\eta_{1} \cos \left(\frac{4 \pi}{L} x\right)-\eta_{2} \cos \left(\frac{8 \pi}{L} x\right)+\cdots\right] .
$$

In the following we truncate the series after two terms and treat the first- and second-harmonic amplitudes $\eta_{1}$ and $\eta_{2}$ of the density as variational parameters that minimize the energy (24),

$$
\begin{aligned}
& \frac{\partial \varepsilon_{1}}{\partial \eta_{1}}=0, \\
& \frac{\partial \varepsilon_{1}}{\partial \eta_{2}}=0 .
\end{aligned}
$$

This truncation, which considerably simplifies the numerics, reproduces the density profile $\rho_{1}(x)$ and variational energy $\varepsilon_{1}$ with the chemical potential taken as $\mu_{1}=1 / L^{2}$, in the case of weak interaction strengths of experimental relevance, as further discussed later on.

Figure 3 shows the resulting density profile for a tilt angle $\alpha=3 \pi / 20$ and as a function of the effective interaction strength $g_{1}$. For $g_{1}=0$ the density distribution is of course uniform, but as $g_{1}$ is increased, the inhomogeneity in the

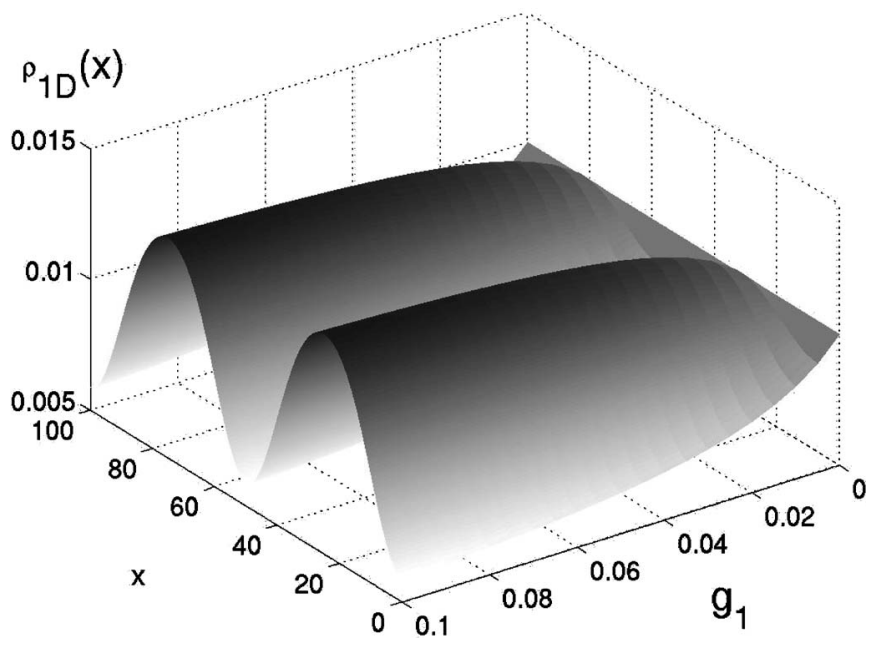

FIG. 3. One-dimensional density distribution as a function of $z$ and $g_{1}$ for $\alpha=3 \pi / 20$.

interaction forces the molecules to increasingly bunch together in the two regions of lower interaction energy. This can be seen by comparing the density distribution of Fig. 3 with the spatial dependence of $V_{c}\left(z_{c}\right)$ in Fig. 2(b). The presence of inhomogenous ground states in ring geometries has previously been found for bosons with homogenous interaction strength $[33,34]$.

In addition to the tilt angle $\alpha$, the ground-state properties of the system depend on two dimensionless parameters only: the aspect ratio $L=L_{0} / a_{0}$ between the characteristic size of the transverse wave function and the storage ring length and the interaction constant $g_{1}$, as defined in Eq. (25). We found numerically the existence of a region in parameter space with no stable solution of Eq. (30). This appears to be due to the attractive contribution of the dipole interaction in some regions along the ring, which for strong enough interaction strengths may destabilize the Thomas-Fermi ground state.

Figure 4 shows the domain of stability of the ThomasFermi ground state (a) for a fixed aspect ratio $L$ and varying tilt angle $\alpha$ and (b) for a fixed tilt angle and varying effective length $L$. Increasing the tilt angle causes the ground state to become unstable for lower values of the interaction strength $g_{1}$, which is inversely proportional to the number of particles. Higher values of $\alpha$ cause the attractive contribution to $V_{c}(z)$ to become stronger, thus localizing the fermionic atoms or molecules into a region of decreasing size. This bunching gives rise in turn to a density profile with a high gradient, eventually causing the mean-field treatment to break down.

Figure 4(b) shows that larger values of the effective length result in the Thomas-Fermi ground state becoming unstable for lower values of $g_{1}$. Equivalently, for a given transverse trap frequency, a larger ring size increases the number of molecules that can be stored, as would be intuitively expected.

One important question is to determine whether the instability of the Thomas-Fermi ground state is simply a result of the truncation scheme (29) and whether increasing the number of terms in the expansion would improve things in case the density gradients along the ring become significant. While increasing the number of Fourier components does 

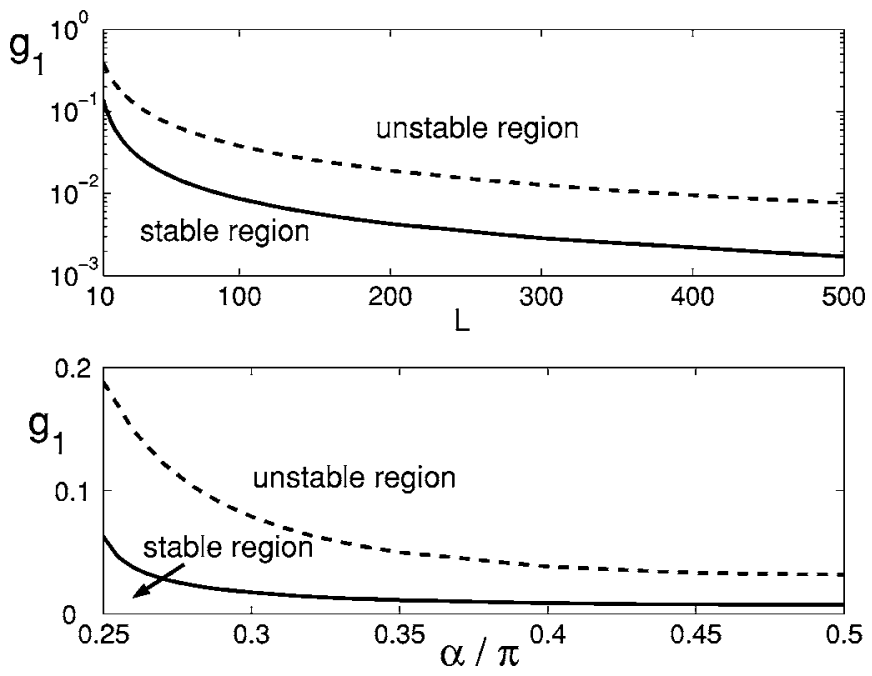

FIG. 4. Thomas-Fermi stability diagram for the one-dimensional case. Critical dimensionless interaction strength as a function of (a) $L$ for $\alpha=2 \pi / 5$ and (b) as a function of $\alpha$ for $L=100$. The dashed line shows the critical interaction strength as a function of (a) $L$ for $\alpha=2 \pi / 5$ and (b) $\alpha$ for $L=100$ for a local interaction potential $V_{r}\left(z_{r}\right)=\delta\left(z_{r}\right)$.

help to better determine the stability domain, the instability appears to be real, as can be seen by considering the simplified model of a local two-body interaction

$$
V_{\mathrm{dd}, \|}\left(z_{c}, z_{r}\right)=V_{c}\left(z_{c}\right) \delta\left(z_{r}\right) .
$$

In that case Eq. (28) reduces to

$$
\rho_{1}^{2}(x)+\frac{g_{1}}{3} V_{c}(x) \rho_{1}(x)=\mu_{1} .
$$

Equation (28) is quadratic in $\rho_{1}$ and can be solved with the chemical potential $\mu_{1}$ as a parameter. We find that, for $\mu_{1} \geqslant 0$, the normalization constraint (23) can only be satisfied for certain values of the parameters $\alpha, L$, and $g_{1}$.

The electric-dipole moment of a typical heteronuclear dimer is of the order of $1 \mathrm{D}$, and its mass is of the order of $\sim 100 \mathrm{~g} / \mathrm{mol}$. Tight transverse trapping yields $a_{0} \sim 1 \mu \mathrm{m}$. For a storage ring length of $500 \mu \mathrm{m}$ these figures imply that a critical interaction strength is $g_{1} \simeq 2 \times 10^{-3}$, a value found in Fig. 5 at $L \sim 500$. As a consequence of the scaling of $g_{1}$ with $1 / N$, it follows that the Thomas-Fermi ground state is stable for particle numbers around $N \sim 10^{2}-10^{4}$ and unstable for smaller samples.

The strength of the magnetic-dipole moment for paramagnetic molecules such as $\mathrm{CrRb}$ or atoms with a permanent magnetic moment is of the order of $\sim 10 \mu_{B}$. This results in an effective one-dimensional interaction strength $g_{1}$ $\sim 10^{-6}-10^{-8}$, yielding a similar stability regime to the case of permanent electric dipoles treated above.

\section{THREE-DIMENSIONAL CASE}

When the transverse trapping potential of the ring is weak compared to the two-body interaction energy, the system is truly three dimensional and the wave function of the fermi-
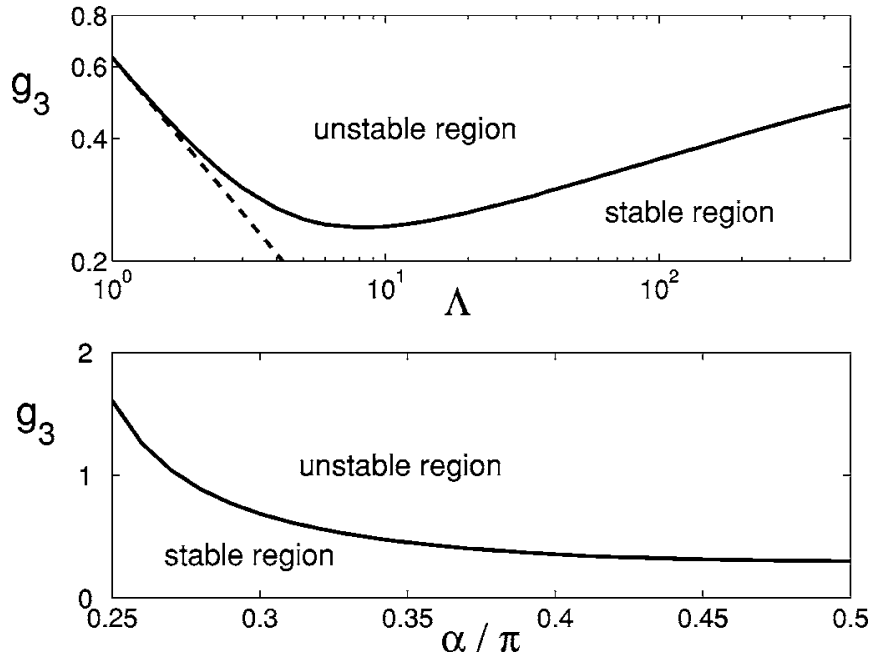

FIG. 5. Stability diagram of the Thomas-Fermi ground state for the three-dimensional regime: (a) Critical interaction strength $g_{3}(\Lambda)$ for $\alpha=2 \pi / 5$; (b) $g_{3}(\alpha)$ for $\Lambda=100$.

ons can no longer be taken as the single-particle ground state. We instead use a Gaussian profile whose width is treated as a variational parameter.

The three-dimensional Thomas-Fermi energy functional is

$$
E=T_{3}+V_{\text {trap }}+V_{\text {int }},
$$

where the kinetic energy $T_{3}$ is given by

$$
T_{3}=\gamma \int n^{5 / 3}(\vec{r}) d^{3} r
$$

$n(\vec{r})$ is the single-particle density obeying the normalization condition

$$
\int n(\vec{r}) d^{3} r=N,
$$

$N$ is the total number of particles, and

$$
\gamma=\left(\frac{3}{\pi}\right)^{2 / 3} \frac{3 \pi^{2} \hbar^{2}}{10 m} .
$$

A key difference between Eq. (35) and the one-dimensional expression (13) is the scaling of the kinetic energy with density, a property with well-known consequences such as the appearance of a Tonk-Girardeau gas in dilute quantumdegenerate bosonic systems.

The second term in the Thomas-Fermi energy functional $E$ is the transverse trapping potential of the storage ring,

$$
V_{\text {trap }}=\frac{1}{2} m \omega_{\perp}^{2} \int\left(x^{2}+y^{2}\right) n(\vec{r}) d^{3} r,
$$

and the third term is the effective dipole-dipole interaction

$$
V_{\mathrm{int}}=\frac{1}{2} \int V_{\mathrm{dd}}\left(\vec{r}, \overrightarrow{r^{\prime}}\right) n(\vec{r}) n\left(\vec{r}^{\prime}\right) d^{3} r d^{3} r^{\prime} .
$$

As in the one-dimensional case, we factorize the density according to 


$$
n(\vec{r})=\left|\Phi_{\perp}\left(\vec{r}_{\perp}\right)\right|^{2} n(z),
$$

but the transverse wave function is now a Gaussian

$$
\Phi_{\perp}\left(\vec{r}_{\perp}\right)=\frac{1}{\sqrt{\pi \Delta r_{\perp}}} \exp \left(-\frac{r_{\perp}^{2}}{2\left(\Delta r_{\perp}\right)^{2}}\right)
$$

whose width $\Delta r_{\perp}$ is taken as a variational parameter. With this ansatz the normalization condition (40) becomes

$$
\int_{0}^{L_{0}} n(z) d z=N
$$

Inserting Eqs. (40) and (41) into the kinetic energy (35) gives

$$
T_{3}=\frac{\gamma}{\left(\Delta r_{\perp}\right)^{4 / 3}} \int n^{5 / 3}(z) d z .
$$

Similarly the transverse trapping energy and the interaction energy become

$$
V_{\text {trap }}=\frac{N}{2} m \omega_{\perp}^{2}\left(\Delta r_{\perp}\right)^{2}
$$

and

$$
V_{\mathrm{int}}=\frac{|\mu|^{2}}{8 \pi \epsilon_{0}\left(\Delta r_{\perp}\right)^{3}} \int_{0}^{L_{0}} d z d z^{\prime} V_{\mathrm{eff}}\left(\frac{z}{\Delta r_{\perp}}, \frac{z^{\prime}}{\Delta r_{\perp}}\right) n(z) n(z) .
$$

We introduce for convenience the dimensionless length

$$
x=\frac{z}{r_{\text {eff }}},
$$

where $r_{\text {eff }}=N^{1 / 6} \sqrt{\hbar / m \omega_{\perp}}$, the dimensionless density

$$
\rho_{3}(x)=\left(\frac{r_{\mathrm{eff}}}{N}\right) n(z),
$$

with

$$
\int_{0}^{\Lambda} \rho_{3}(x) d x=1
$$

and the dimensionless variational parameter

$$
\ell=\Delta r_{\perp} / r_{\text {eff }} .
$$

This scaling results in the dimensionless energy functional

$$
\begin{aligned}
\varepsilon_{3}= & \frac{E_{3} r_{\mathrm{eff}}^{2}}{\gamma N^{5 / 3}}=\frac{1}{\ell^{4 / 3}} \int_{0}^{\Lambda} \rho_{3}(x)^{5 / 3} d x+\beta \ell^{2} \\
& +\frac{1}{2} \frac{g_{3}}{\ell^{3}} \int_{0}^{\Lambda} V_{\mathrm{eff}}\left(\frac{x}{\ell}, \frac{x^{\prime}}{\ell}\right) \rho_{3}(x) \rho_{3}\left(x^{\prime}\right) d x d x^{\prime},
\end{aligned}
$$

where $\Lambda=L_{0} / r_{\text {eff }}, \beta=(\pi / 3)^{2 / 3}\left(5 / 3 \pi^{2}\right)$, and the dimensionless interaction strength is

$$
g_{3}=\frac{|\mu|^{2}}{4 \pi \epsilon_{0} \gamma} \sqrt{\frac{m \omega_{\perp}}{\hbar}} N^{1 / 6} .
$$

In marked contrast with the quasi-one-dimensional case, the two-body interaction now dominates over the kinetic en- ergy in the limit of high densities, with a scaling reminiscent of the corresponding three-dimensional dilute Bose gas with hard-sphere interaction [35].

As in the quasi-one-dimensional case we exploit the periodicity of the longitudinal density distribution to justify a variational ansatz of the form

$$
\rho_{3}(x)=\frac{1}{\Lambda}\left[1-\eta_{3} \cos (4 \pi x / \Lambda)\right],
$$

where we now keep only the first Fourier component for numerical simplicity. The ground-state density is determined by optimizing the energy functional $\varepsilon_{3}$ with respect to the free parameters,

$$
\begin{gathered}
\frac{d \varepsilon_{3}}{d \ell}=0, \\
\frac{d \varepsilon_{3}}{d \eta_{3 D}}=0 .
\end{gathered}
$$

Figure 5 shows the resulting Thomas-Fermi stability diagram as a function of $\Lambda$ for constant tilt angle $\alpha=2 \pi / 5$ and as a function of $\alpha$ for a fixed effective length $\Lambda=100$. For a fixed effective length, a larger value of the tilt angle $\alpha$ increases the range of effective strengths, resulting in a valid Thomas-Fermi ground state. The system becomes unconditionally stable for $\alpha \leqslant \arcsin (1 / \sqrt{3}) \sim 35^{\circ}$ since the dipoledipole interaction is then repulsive along the whole ring. The stability diagram for a fixed angle $\alpha=2 \pi / 5$ shown in Fig. 5 (a) is more complex, with a minimum of $g_{3}$ around $\Lambda=8$.

For the same atomic or molecular parameters as in the quasi-one-dimensional case, but with a weaker trap of width $10 \mu \mathrm{m}$, we find that $N \leqslant 4 \times 10^{4}$ for the case of fermions with permanent electric dipoles and $\alpha=2 \pi / 5$ and $N \leqslant 2$ $\times 10^{4}$ for $\alpha=\pi / 4$. These values indicate that it should be fairly easy to investigate physics beyond mean-field theory for ultracold fermions in ring geometries.

Figure 6 shows the relative contributions to the energy functional as functions of the effective length $\Lambda$ for fixed tilt angle $\alpha=2 \pi / 5$. The interaction energy $V_{\text {int }}$ increases and the attractive interaction energy decreases with $\Lambda$, but the total energy is always dominated by the kinetic energy, and as a result the critical value of the effective strength increases.

Figure 5 shows that the critical interaction strength increases for small values of $\Lambda$, even though the (attractive) interaction energy decreases; see Fig. 6. This behavior can be intuitively understood in terms of a simplified analysis that takes the relative part of the interaction to be short ranged,

$$
V_{R}=\delta\left[\left(x-x^{\prime}\right) / \ell\right] .
$$

We consider for illustration the stability of the ThomasFermi ground state for a tilt angle $\alpha>\arcsin (1 / \sqrt{3})$ and thus take the interaction to be attractive. Inserting the ansatz (53) into Eq. (49) gives

$$
\varepsilon_{3} \approx \frac{1}{\ell^{4 / 3}}\left(\frac{1}{\Lambda}\right)^{2 / 3}+\beta \ell^{2}-g_{3} \kappa \frac{1}{\ell^{2}}
$$

where $\kappa$ is a coefficient that includes numerical factors and the value of the interaction energy. For the value $\ell_{m}$ that 


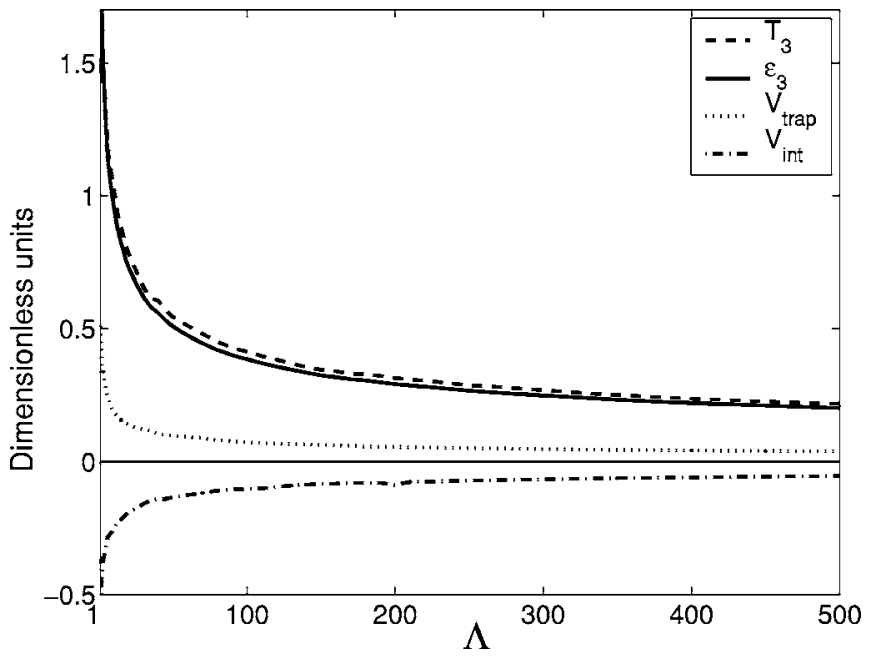

FIG. 6. Relative contributions to the energy functional $\varepsilon_{3}$ as functions of $\Lambda$ for $\alpha=2 \pi / 5$ for the critical interaction strength $g_{3}$, illustrating the distribution of energies at the border of the instability region.

minimizes Eq. (54) the effective interaction strength will satisfy the condition

$$
\left[\frac{2}{3}\left(\frac{1}{\Lambda}\right)^{2 / 3}-2 \frac{\kappa g_{3}}{\ell_{m}^{2 / 3}}\right]^{3}=\beta^{3} \ell_{m}^{10} .
$$

For $\ell_{m}$ to be positive we have then

$$
\left(\frac{1}{\Lambda}\right)^{2 / 3}>\frac{3}{2} \frac{\kappa_{1} g_{3}}{\ell_{m}^{2 / 3}}
$$

where $\kappa_{1}$ is a positive constant. Equation (55) can furthermore be rearranged to give

$$
\left[\frac{2}{3}\left(\frac{1}{\Lambda}\right)^{2 / 3}-\beta \ell_{m}^{10 / 3}\right]^{3}=\frac{\left(2 \kappa g_{3}\right)^{3}}{\ell_{m}^{2}},
$$

which gives, for $\ell_{m}>0$,

$$
\left(\frac{1}{\Lambda}\right)^{2 / 3}>\kappa_{2} \ell_{m}^{10 / 3}
$$

where $\kappa_{2}$ is another positive constant. Upon combining Eqs. (55) and (58) we finally have

$$
g_{3}<\kappa_{3} \Lambda^{-4 / 5},
$$

where the constant $\kappa_{3}$ is also positive. It follows from that simplified model that increasing $\Lambda$ causes the critical value of the $g_{3}$ to decrease, which explains qualitatively its behavior in the regime of small effective lengths. The upper bound given by Eq. (59) is shown as a dashed line in Fig. 4(a). For small values of $\Lambda$ the critical interaction strength is seen to scale as $\Lambda^{-4 / 5}$, in agreement with the simplified analysis. This indicates that for small values of $\Lambda$ the short-range properties of the interaction dominate the stability properties of the system, which remains stable as long as the different contributions to the energy functional (51) can balance each other.

\section{CONCLUSIONS}

In this paper we have studied the Thomas-Fermi ground state of a system of spin-polarized fermions interacting via the dipole-dipole interaction in a circular waveguide geometry and subjected to an additional static external field. That field results in an inhomogeneous interaction with a periodicity of $\pi$ in the center-of-mass frame, and due to the tunable inhomogeneity of the interaction, it can be attractive in certain domains along the ring.

We find that the Thomas-Fermi ground-state density distribution of the atomic or molecular fermions is then likewise periodic, with a large fraction of particles residing in the region of minimum energy. For small rings and large interaction strengths the particles are then localized in diametrically opposite regions. However, in the quasi-onedimensional case there is a lower bound to the number of particles for which that ground state is stable and an upper bound in three-dimensional case. The different scaling in these two situations is a direct consequence of the scaling of the kinetic energy of the fermionic sample with density.

The instability of the Thomas-Fermi ground state, and the concomitant breakdown of the Thomas-Fermi theory, signals the presence of nontrivial quantum correlations in the true many-body ground state, and a treatment beyond mean-field theory is needed to deal with that situation. It is known, for instance, that in quasi-one-dimensional systems and for low particle densities fermionic systems can under appropriate circumstances undergo a Wigner crystallization [36], in analogy with the situation for trapped ions; see e.g. [37-39].

We finally note that in the case of large rings [21], the system is also far from the Thomas-Fermi situation described in this paper. A quantum dynamical treatment using wavepacket methodologies that is more appropriate in this case will be described in a subsequent paper.

\section{ACKNOWLEDGMENTS}

This work is supported in part by the U.S. Office of Naval Research, by the National Science Foundation, by the U.S. Army Research Office, by the Joint Services Optics Program, and by the National Aeronautics and Space Administration.
[1] E. A. Donley, N. R. Claussen, S. T. Thompson, and C. E. Wieman, Nature (London) 417, 529 (2002).

[2] M. Greiner, C. A. Regal, and D. Jin, Nature (London) 426, 537 (2003).
[3] M. Zwierlein et al., Phys. Rev. Lett. 91, 250401 (2003).

[4] S. Jochim et al., Science 301, 2101 (2003).

[5] C. A. Stan et al., Phys. Rev. Lett. 93, 143001 (2004).

[6] S. Inouye et al., Phys. Rev. Lett. 93, 183201 (2004). 
[7] A. J. Kerman et al., Phys. Rev. Lett. 92, 033004 (2004). 92, 153001 (2004). T. Bergeman et al., Eur. Phys. J. D 31, 179 (2004).

[8] R. Folman et al. Adv. At., Mol., Opt. Phys. 48, 263 (2002). J. Reichel, Appl. Phys. B: Lasers Opt. 74, 469 (2002).

[9] Atom Chips, edited by C. Henkel, J. Schmiedmayer, and C. Westbrook Eur. Phys. J. D 35, 1 (2005).

[10] J. F. Clauser, Physica B \& C 151, 262 (1988).

[11] S. Gupta et al., Phys. Rev. Lett. 95, 143201 (2005).

[12] A. S. Arnold and E. Riis, J. Mod. Opt. 49, 959 (2002); A. S. Arnold, J. Phys. B 37, L29 (2004); A. S. Arnold, C. S. Garvie, and E. Riis, e-print cond-mat/0506142.

[13] A. Hopkins, B. Lev, and H. Mabuchi, Phys. Rev. A 70, 053616 (2004).

[14] J. A. Sauer, M. D. Barrett, and M. S. Chapman, Phys. Rev. Lett. 87, 270401 (2001).

[15] S. Wu, W. Rooijakkers, P. Striehl, and M. Prentiss, Phys. Rev. A 70, 013409 (2004).

[16] M. Tscherneck et al., J. Phys.: Conf. Ser. 19, 66 (2005).

[17] Ultracold Dipolar Molecules, edited by J. Doyle, B. Friedrich, R. V. Krems, and F. Masnou-Seeuws Eur. Phys. J. D 31, 149 (2004).

[18] S. Kotochigova, P. S. Julienne, and E. Tiesinga, Phys. Rev. A 68, 022501 (2003).

[19] See, e.g., F. M. H. Crompvoets, R. T. Jongma, H. L. Bethlem, Andr J. A. van Roij, and G. Meijer, Phys. Rev. Lett. 89, 093004 (2002); M. R. Tarbutt, H. L. Bethlem, J. J. Hudson, V. L. Ryabov, V. A. Ryzhov, B. E. Sauer, G. Meijer, and E. A. Hinds, ibid. 92, 173002 (2004); F. M. H. Crompvoets, H. L. Bethlem, J. Küpper, A. J. A. van Roij, and G. Meijer, Phys. Rev. A 69, 063406 (2004).

[20] T. Junglen, T. Rieger, S. A. Rangwala, P. W. H. Pinkse, and G. Rempe, Phys. Rev. Lett. 92, 223001 (2004).

[21] F. M. H. Crompvoets et al., Nature (London) 411, 6834
(2001).

[22] K. Goral, B.-G. Englert, and K. Rzazewski, Phys. Rev. A 63, 033606 (2001).

[23] S. Giovanazzi and D. H. J. O’Dell, Eur. Phys. J. D 31, 439 (2004).

[24] K. Goral, M. Brewczyk, and K. Rzazewski, Phys. Rev. A 67, 025601(R) (2003).

[25] J. R. Bochinski, E. R. Hudson, H. J. Lewandowski, G. Meijer, and Jun Ye, Phys. Rev. Lett. 91, 243001 (2003).

[26] L. Santos, G. V. Shlyapnikov, P. Zoller, and M. Lewenstein, Phys. Rev. Lett. 85, 1791 (2000).

[27] S. Yi, L. You and H. Pu, Phys. Rev. Lett. 93, 040403 (2004).

[28] D. H. J. O’Dell, S. Giovanazzi, and C. Eberlein, Phys. Rev. Lett. 92, 250401 (2004); K. Goral and L. Santos, Phys. Rev. A 66, 023613 (2002).

[29] L. Spruch, Rev. Mod. Phys. 63, 151 (1991).

[30] E. H. Lieb, Rev. Mod. Phys. 53, 603 (1981).

[31] M. D. Girardeau and M. Olshanii, Phys. Rev. A 70, 023608 (2004).

[32] M. Girardeau, J. Math. Phys. 1, 516 (1960).

[33] R. Kanamoto, H. Saito, and M. Ueda, Phys. Rev. A 67, 013608 (2003).

[34] G. M. Kavoulakis, Phys. Rev. A 67, 011601(R) (2003).

[35] V. I. Yukalov and M. D. Girardeau, Laser Phys. Lett. 2, 375 (2005).

[36] P. A. Martin and F. Rothen, Many-body Problems and Quantum Field Theory (Springer, Berlin, 2002).

[37] F. Diedrich, E. Peik, J. M. Chen, W. Quint, and H. Walther, Phys. Rev. Lett. 59, 2931 (1987).

[38] D. J. Wineland, J. C. Bergquist, W. M. Itano, J. J. Bollinger, and C. H. Manney, Phys. Rev. Lett. 59, 2935 (1987).

[39] M. Drewsen, C. Brodersen, L. Hornekaer, J. S. Hangst, and J. P. Schiffer, Phys. Rev. Lett. 81, 2878 (1998). 